\title{
Erratum to: Closure of circular arc cracks under general loading: effects on stress intensity factors
}

\author{
Elizabeth Ritz • David D. Pollard
}

Published online: 10 August 2011

(C) Springer Science+Business Media B.V. 2011

\section{Erratum to: Int J Fract (2011) 167(1):3-14 DOI 10.1007/s10704-010-9511-8}

Due to an unfortunate turn of events, this article has been published with an erroneous version of Fig. 6 and two errors in the values of the second paragraph as well as one error in the text of paragraph three of Sect. 5 "Arc crack closure under uniaxial tension". Please find below the correct values and text part of the second and third paragraph of Sect. 5 and the correct Fig. 6 that should be regarded by the reader as the final versions.

Figure 6 shows displacement fields normalized by the magnitude of the maximum displacement within that field. The initial and exaggerated final crack geometries are also shown in Fig. 6 in order to illustrate the deformation of the crack surfaces. Note that in general, displacements within the concavity of the arc crack have greater magnitudes than those in the surrounding material. Although the displacement fields in Fig. 6

The online version of the original article can be found under doi:10.1007/s10704-010-9511-8.

E. Ritz $(\varangle) \cdot$ D. D. Pollard

Department of Geological and Environmental Sciences, Stanford University, 450 Serra Mall, Building 320,

Stanford, CA 94305, USA

e-mail: 1ritz@stanford.edu

URL: http://pangea.stanford.edu/research/geomech/ are plotted with similar vector lengths, the magnitude of the maximum displacement differs both with arc crack half-angle $\alpha$ and loading orientation $\omega$. In comparing the examples of arc cracks with $\alpha=45^{\circ}$ and $\alpha=90^{\circ}$, the lesser half angle yields lesser displacement discontinuities. For all arc crack half angles, as $\omega$ increases and the direction of uniaxial tension becomes more parallel to the arc crack chord, the magnitude of maximum displacement decreases. As the loading orientation $\omega$ increases from $0^{\circ}$ to $90^{\circ}$, the maximum displacement discontinuity magnitude plotted decreases 80.6 and $58.0 \%$ for arc cracks with $\alpha=45^{\circ}$ and $\alpha=$ $90^{\circ}$, respectfully. Additionally, the rate of change of the maximum magnitude increases as the loading orientation $\omega$ increases from $0^{\circ}$ to $90^{\circ}$.

Somewhat different behaviors in normal displacement discontinuity are observed for cracks with half angles greater than and less than $77.2^{\circ}$. For cracks with half angles $\alpha<77.2^{\circ}$, as the orientation of the applied stress increases from $\omega=0^{\circ}$, the crack will eventually close, initially at the right tip. For example, consider the arc crack $\alpha=45^{\circ}$; the corresponding closing conditions are shown in Fig. 4a, displacement fields for specific loading orientations are shown in Fig. 6a, and normal displacement discontinuity distributions are shown in Fig. 7a. The crack is completely open for loading angles of $\omega=0^{\circ}$ and $30^{\circ}$, while the right crack tip is closed for $\omega=45^{\circ}$ and $60^{\circ}$. Numerical experiments show that partial closure occurs for this particular arc crack geometry with a loading orientation greater than 
$\omega=34.4^{\circ}$. Loading angles of the remote uniaxial tension greater than $\omega=34.4^{\circ}$ cause the material on the concave side of the arc crack to displace toward the right crack tip, forcing the upper crack surface to come into contact with the lower surface. As $\omega$ increases and the direction of uniaxial tension becomes more nearly parallel to the arc crack chord, the right crack tip opens and the material on the convex side of the arc crack contracts and moves toward the center of the crack, causing contact (Fig. 6a). For an arc crack with half angle $\alpha=45^{\circ}$, the right crack tip opens for loading orientations $\omega>67.7^{\circ}$. As the orientation angle $\omega$ increases to $90^{\circ}$, the closed section of the crack continues to shift away from the right tip until it is centered at the middle of the arc crack at $\omega=90^{\circ}$.
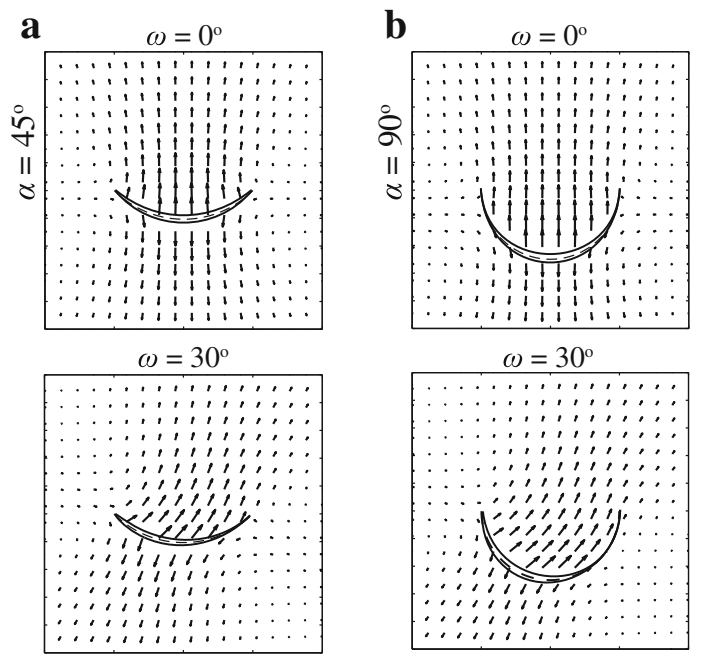

$\omega=45^{\circ}$

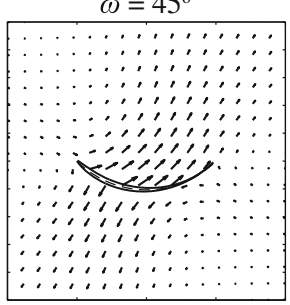

$\omega=60^{\circ}$

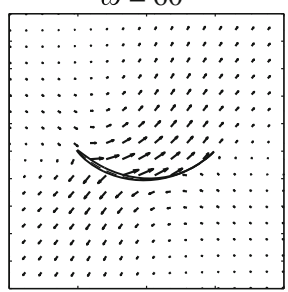

$\omega=90^{\circ}$
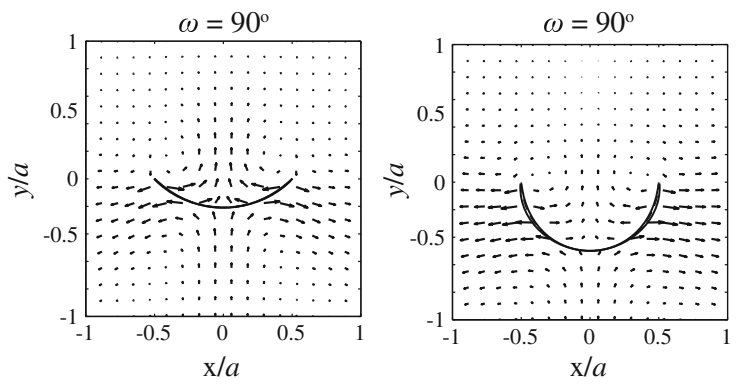

Fig. 6 Displacement fields due to remote uniaxial tension, normalized by the magnitude of the maximum displacement within that field. The initial crack geometry is shown by the dashed curve, while the distorted crack geometry is shown by two solid curves. Crack surface distortion is magnified 600 times. Displacement fields for various orientation angles $\omega$ of the circular arc crack with half angle $\mathbf{a} \alpha=45^{\circ}$ and $\mathbf{b} \alpha=90^{\circ}$ 